\title{
The search for Population III stars
}

\author{
Sperello di Serego Alighieri ${ }^{1}$, Jaron Kurk ${ }^{2}$, Benedetta Ciardi ${ }^{3}$, \\ Andrea Cimatti ${ }^{4}$, Emanuele Daddi ${ }^{5}$ and Andrea Ferrara ${ }^{6}$ \\ ${ }^{1}$ INAF - Osservatorio Astrofisico di Arcetri, Largo E. Fermi 5, Firenze, Italy \\ email: sperello@arcetri.astro.it \\ ${ }^{2}$ Max Planck Institut für Astronomie. Königstuhl 17, Heidelberg, Germany \\ ${ }^{3}$ Max Planck Institut für Astrophysik, Karl Schwarzschild Str. 1, Garching, Germany \\ ${ }^{4}$ Università di Bologna, Via Ranzani 1, Bologna, Italy \\ ${ }^{5} \mathrm{CEA} /$ Saclay, Gif sur Yvette, France \\ ${ }^{6}$ SISSA, Via Beirut 4, Trieste, Italy
}

\begin{abstract}
Population III stars, the first generation of stars formed from primordial Big Bang material with a top-heavy IMF, should contribute substantially to the Universe reionization and they are crucial for understanding the early metal enrichment of galaxies. Therefore it is very important that these objects, foreseen by theories, are detected by observations. However PopIII stars, searched through the HeII 1640Å line signature, have remained elusive. We report about the search for the HeII line in a galaxy at $\mathrm{z}=6.5$, which is a very promising candidate. Unfortunately we are not yet able to show the results of this search. However we call attention to the possible detection of PopIII stars in a lensed HII dwarf galaxy at $\mathrm{z}=3.4$, which appeared in the literature some years ago, but has been overlooked.
\end{abstract}

Keywords. cosmology: observation, galaxies: formation, stars: chemically peculiar

\section{Introduction}

Theoretical models foresee that the first generation of stars, forming from primordial Big Bang material, should have unusually massive stars (a top-heavy IMF), with masses up to about $500 M_{\odot}$ (Schneider et al. 2002). These stars are called Population III (PopIII) stars, and at least some of them are expected to quickly release metals in the interstellar medium (ISM); therefore later generations would soon be metal enriched. The metallicity threshold in the ISM for producing PopIII stars should be around $10^{-5}-10^{-4} Z_{\odot}$. The very massive PopIII stars would then produce a short phase of unusually hard and strong UV radiation $\left(T_{\text {eff }} \sim 100000 K\right)$, resulting in a specific line emission signature (Schaerer 2002). The most prominent and unique emission line is expected to be HeII $1640 \AA$, which can become as strong as $1 / 3$ of Lyman $\alpha$, making it observable with current 8 $10 \mathrm{~m}$ telescopes in the highest redshift galaxies known (Scannapieco et al. 2003).

The detection of PopIII stars would be extremely important for understanding the early chemical evolution of galaxies, and because they should be crucial contributors to the reionization of the Universe (Ciardi et al. 2003). PopIII stars have however remained elusive: searches for HeII $1640 \AA$ through stacking of spectra of Lyman $\alpha$ emitting galaxies (Dawson et al. 2004 and Ouchi et al. 2008), through deep spectroscopy of an individual galaxy (Nagao et al. 2005), or through dual (Ly $\alpha$ and HeII) narrow-band imaging (Nagao et al. 2008) have failed. Jimenez \& Haiman (2006) have ascribed to PopIII stars the HeII $1640 \AA$ line detected in the composite spectrum of $\sim 1000$ Lyman break galaxies at $z \sim 3$, but the line is only about $1 / 10$ of Lyman $\alpha$ and is considerably broader: it could therefore be attributed to a stellar wind feature associated with massive WR stars (Shapley et al. 
2003). The upper limits to the star formation rate (SFR) for PopIII stars, which can be set from these negative results are now quite close to the rates expected from the models of Tornatore et al. (2007) (see the contribution by T. Nagao to these Proceedings).

We report about the search for the HeII $1640 \AA$ line in a galaxy at $\mathrm{z}=6.5$ and about the possible detection of PopIII stars in a lensed dwarf HII galaxy at $\mathrm{z}=3.4$, which has appeared in the literarure (Fosbury et al. 2003), but has so far been overlooked.

\section{The search for PopIII stars in KCS 1166}

KCS 1166 is a Lyman $\alpha$ emitting galaxy at $\mathrm{z}=6.518$, discovered with slitless spectroscopy in the atmospheric window at $\lambda \sim 9100 \AA$ (Kurk et al. 2004). In this object the Lyman $\alpha$ line is clearly asymmetric, being steeper on the blue side, has a very large equivalent width, at least $80 \AA$ in the rest frame, and a luminosity of $1.1 \times 10^{43} \mathrm{erg} \mathrm{s}^{-1}$. No continuum is detected at shorter wavelengths, while it is present redward of the line. These characteristics and the fact that the HeII $1640 \AA$ line is expected in a region of the $\mathrm{J}$-band relatively free of sky emissions make KCS 1166 a very good candidate for the search of PopIII stars.

We have therefore carried out near IR spectroscopy in the J-band of KCS 1166, using SINFONI, an Integral Field Spectrograph at the VLT (Bonnet et al. 2004), with a total on-source exposure time of 9 hours, equally spread over 3 consecutive nights (Fig. 1). Thanks to the excellent efficiency in the J-band and the lack of $\mathrm{OH}$ Suppressor and of slit losses of SINFONI, we estimate that our observations of KCS 1166 should be a factor of about 1.5 more efficient in detecting the eventual presence of the HeII $1640 \AA$ line than those of SDF J132440.6+273607 at $\mathrm{z}=6.3$ by Nagao et al. (2005), although we had a slightly shorter exposure time.

However at the time of this Conference we are yet unable to report about the results of our observations, since the data reduction, which is rather complicated for an Integral Field Spectrograph like SINFONI, is not yet finished.
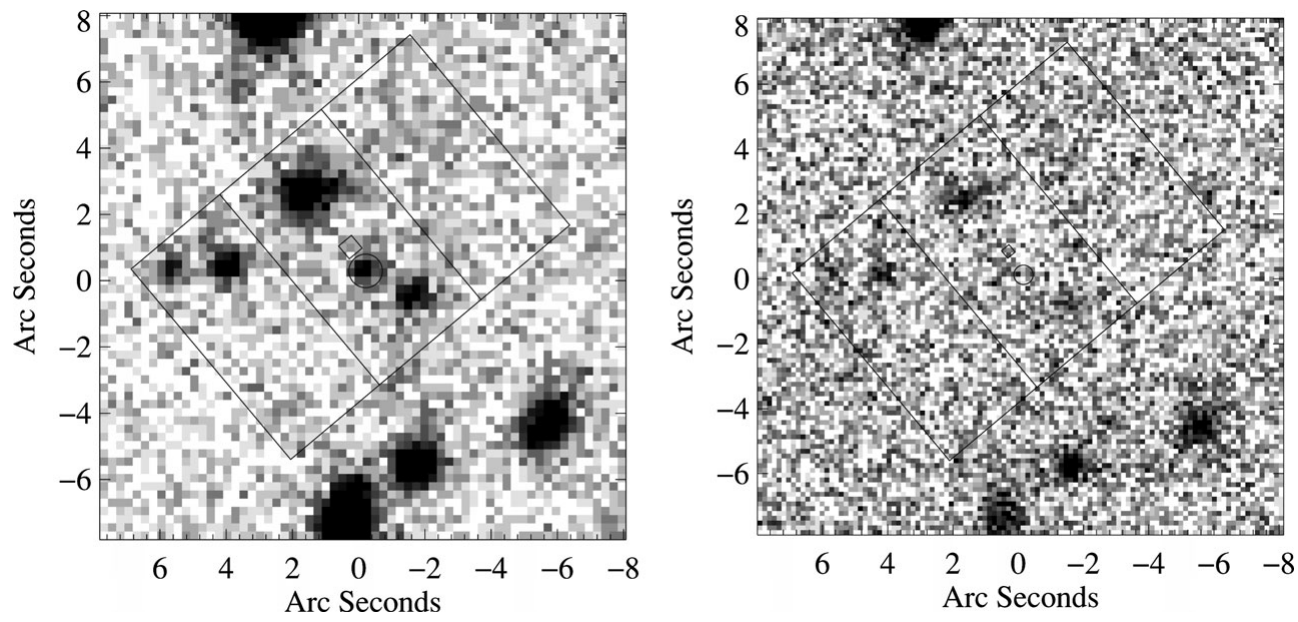

Figure 1. FORS z-band (left) and ISAAC J-band (right) images of the field of KCS 1166. The large boxes mark the two squared areas covered by the Integral Field of view of SINFONI in the two observing positions, shifted by half of the field size to improve the sky subtraction and to leave KCS 1166 in the overlapping area observed for the whole observing time. The galaxy is marked by a small circle and is clearly visible only in the $\mathrm{z}$-band, which contains the Lyman $\alpha$ line. 


\section{The possible detection of PopIII stars in the Lynx arc}

We take this opportunity to draw the attention of the Conference participants and of those interested in PopIII stars to the results obtained by Fosbury et al. (2003) on the Lynx arc, since these could well mark the first detection of PopIII stars, and are completely overlooked by the relevant literature. Although we recommend the interested reader to read the paper by Fosbury et al. directly, we give here a summary of their work.

The Lynx arc is a dwarf HII galaxy at $\mathrm{z}=3.357$, lensed by the $\mathrm{z}=0.570$ cluster $\mathrm{RX}$ J0848+4456, and it has been discovered serendipitously by Holden et al. (2001) during spectroscopic follow-up of the cluster. The HII galaxy is magnified by a factor of about 10 , as evaluated by a detailed analysis of the complex cluster environment from HST WFPC2 images. The unusual emission line spectrum has been thoroughly studied with the LRIS, ESI and NIRSPEC at the Keck telescopes, covering the rest-frame ranges 900-2500 and 3300-5700A. It shows strong and self-absorbed Lyman $\alpha$ line and CIV

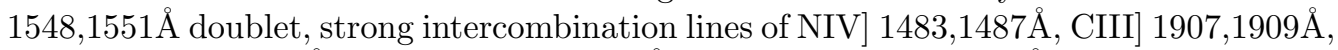

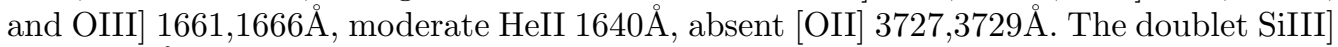
$1883,1892 \AA$ is clearly detected in the ESI spectrum, and is 40 times brighter, relative to $\mathrm{H} \beta$, than foreseen by models with scaled solar abundances. The intercombination lines have a very narrow width $\left(\sigma \sim 30-35 \mathrm{kms}^{-1}\right)$, indicating a small gravitating mass of about $10^{9} M_{\odot}$, typical of a dwarf galaxy. The absence of NV $1240 \AA$ and the weakness of NIII] $1750 \AA$ indicate that the ionizing source is a blackbody, rather than a power law, as it would be expected in case of ionization by an AGN.

The intensity of the continuum observed longward of Lyman $\alpha$ is completely explained by the nebular continuum, as accurately predicted from the observed strength of $\mathrm{H} \beta$. The continuum produced by an instantaneous burst of $10^{7} M_{\odot}$, Salpeter IMF, Z $=0.05 \times Z_{\odot}$, age of 1 to $5 \mathrm{Myr}$ would be seen in the data, if it were present, but would produce an ionizing flux 20 times lower than the one necessary to produce the observed emission lines: this type of continuum, therefore, cannot be the source of ionization.

The photoionization model reproducing all the observed features has a black-body ionizing source with: a) a black-body temperature of $80000 \mathrm{~K}$, much higher than the effective temperature of Galactic compact HII regions, which does not exceed 40000K; b) an ionization parameter $\mathrm{U}=0.1$, also higher than in local HII regions; c) a low nebular metallicity $\mathrm{Z}=0.05 \times Z_{\odot}$. The lack of stellar continuum longward of Lyman $\alpha$, the strong necessary ionizing flux, and the small stellar mass all indicate that the ionizing flux should be produced by fewer than $10^{6}$ hot stars, formed with a top-heavy IMF, and most likely with a metallicity still lower than the nebular one. The substantial overabundance of silicon in the nebula indicate enrichment by past pair instability supernovae, as those resulting from the total disruption of stars with 140-260 $M_{\odot}$ (Hegel \& Woosley 2002).

All these characteristics point strongly to the presence of PopIII stars, some of which might have already exploded to partially enrich the ISM. However in a dwarf galaxy like the Lynx arc, the formation of PopII stars could have been delayed long enough to make the uncontaminated UV signature of PopIII stars detectable at intermediate redshift. In fact this uncontaminated signature of PopIII stars might have a much shorter duration in the massive galaxies, which are the only observable galaxies at high redshift. This might be the reason why PopIII stars have not yet been detected at high redshift, where they have been searched so far, following the obvious paradigm that primordial material is more abundant in the very young Universe.

In a contribution to a conference Schaerer (2004) finds it unlikely that the Lynx arc contains an extremely metal poor cluster, because the ISM metallicity is $1 / 20$ solar and there are no known cases of the stellar metallicity lower than the nebular one, and the 
alternative explanation of an obscured AGN (Binette et al. 2003) seems to be capable of reproducing the observed spectrum. However, it should not be surprising that new phenomena are observed when dealing with a new class of objects, like PopIII stars, and Binette et al. (2003) find it plausible that the stellar metallicity might be lower than the nebular one. Furthermore, of the 5 models presented by Binette et al. (2003) the one that best fits the observed line ratios of the Lynx arc is the hot star (80000 K) model, which is relevant for PopIII and which has only one inconsistent line, Si III] 1883,1892 whose discrepancy could in any case be due to the nucleosynthetic signature of pair instability supernovae, as explained above. The other 4 models also have discrepant Si III] $1883,1892 \AA$, but in addition have at least two more lines inconsistent with the observations. Therefore the most likely explanations for the observed spectrum of the Lynx arc remains the very hot star model expected for PopIII stars.

\section{Final remarks}

Although we are yet unable to report on the results of our observations of the HeII $1640 \AA$ line in KCS 1166, which is a good candidate for PopIII search at $\mathrm{z}=6.518$, it is well possible that PopIII stars have already been detected by Fosbury et al. (2003) in a dwarf star-forming galaxy at $\mathrm{z}=3.357$, thanks to the fortunate combination of the longer uncontaminated PopIII phase in dwarf galaxies and of the opportunity given by the lensing magnification to identify PopIII stars, even if not at their peak activity.

Clearly further detections of PopIII stars, or stringent upper limits, would be extremely important to understand the connection between reionization and metal production, since PopIII stars should be major players on both scenes, and could spoil the simple proportionality between ionizing photons and metals, claimed by some (see e.g. the contribution by A. Ferrara to these Proceedings).

\section{Acknowledgements}

We would like to thank Bob Fosbury and Raffaella Schneider for very useful comments.

\section{References}

Binette, L., Groves, B., Villar-Martin, M., Fosbury, R.A.E. \& Axon, D.J. 2003, A $\& A, 405,975$

Bonnet, H., Abuter, R., Baker, A. et al. 2004, ESO Messenger, 117, 17

Ciardi, B., Ferrara. A. \& White, S.D.M. 2003, MNRAS, 344, L7

Dawson, S., Rhoads, J.E., Malhotra, S. et al. 2004, ApJ, 617,707

Fosbury, R.A.E., Villar-Martin, M., Humphrey, A. et al. 2003, ApJ, 596, 797

Heger, A. \& Woosley, S.E. 2002, ApJ, 567, 532

Holden, B.P., Stanford, S.A., Rosati, P. et al. AJ, 122, 629

Jimenez, R. \& Haiman, Z. 2006, Nature, 441, 120

Kurk, J.D., Cimatti, A., di Serego Alighieri, S. et al. 2004, A\&A, 422, L13

Nagao, T., Motohara, K., Maiolino, R. et al. 2005, ApJ, 631, L5

Nagao, T., Sasaki, S.S., Maiolino, R. et al. 2008, ApJ 680, 100

Ouchi, M., Shimasaku, K., Akiyama, M. et al. 2008, ApJS, 176, 301

Scannapieco, E., Schneider, R. \& Ferrara, A. 2003, ApJ, 589, 35

Schaerer, D. 2002, A\&A, 382, 28

Schaerer, D. 2003, in: M. Plionis (ed.), Multiwavelength Cosmology (Kluwer), p. 219

Schneider, R., Ferrara, A., Natarajan, P., Omukai, K. 2002, ApJ, 571, 30

Shapley, A.E., Steidel, C.C., Pettini, M. \& Adelberger, K.L. 2003, ApJ, 588, 65

Tornatore, L., Ferrara, A. \& Schneider, R. 2007, MNRAS, 382, 945 